\title{
UN CURIEUX CAS DE PSEUDO-PARASITISME PAR ACARIENS DANS UN LIQUIDE CÉPHALO-RACHIDIEN
}

\author{
Par K. SAMSINAK et D. JARRY
}

La littérature médicale fait mention plusieurs fois, depuis le début du siècle, de la découverte d'Acariens dans des urines ou des liquides retirés par ponction. Ces Arthropodes ont été rapportés au genre Tarsonemus, appartenant au sous-ordre des Tarsonemoidea. En l'absence de caractères formels, tant morphologiques qu'écologiques, et, surtout, quant au fait que l'on a souvent affaire à des individus immatures, on s'arrête souvent à la diagnose de Tarsonemus hominis (Dahl). Cette espèce aurait de nombreux synonymes, parmi lesquels : T. floricolus, T. socialis, T. robinii, T. macronychus, T. supinoi.

On s'est demandé si ces Acariens pouvaient avoir un quelconque pouvoir pathogène, allant aux deux extrêmes, soit en leur imputant des accidents nécrotiques fort graves, soit en leur prêtant le rôle de vulgaires corps étrangers entraînés par l'écoulement du liquide à travers une aiguille ou une sonde.

La curieuse observation que nous allons rapporter est précisément en faveur d'une contamination secondaire du prélèvement.

Mme L. M..., demeurant rue de Calais, à Casablanca, est prise, le 4 novembre 1959, d'un malaise brutal avec tendance syncopale et céphalées intenses dans la région cervico-occipitale, alors qu'elle effectuait des travaux ménagers.

Le $\mathrm{D}^{r} \mathrm{R} \ldots$, que nous tenons à remercier ici pour les renseignements qu'il a bien voulu nous donner, a examiné la patiente dans les instants qui ont suivi cet incident. Un état nauséeux ayant succédé à quelques vomissements faciles, une raideur discrète de la nuque et un examen général négatif n'autorisent aucun diagnostic bien précis. Cet examen est d'ailleurs difficile parce que la malade est obèse $(90 \mathrm{~kg}$. pour $1,60 \mathrm{~m}$.) et elle se refuse à une ponction lombaire. Le praticien, craignant une hémorragie méningée, prescrit un traitement symptomatique. Au bout de quelques jours et devant la persistance de céphalées intenses, Mme L... se résout à la ponction. Celle-ci, en date du 9 septembre 1959, donne les 
résultats suivants: liquide eau de roche, 118 hématies et 56 globules blancs par $\mathrm{mm}^{3}$ (dont $82 \%$ de polynucléaires et $18 \%$ de lymphocytes), enfin $0,18 \mathrm{~g}$. d'albumine par litre.

Le 14, une nouvelle ponction lombaire est pratiquée. Pour un résultat à peu près superposable, elle offre à considérer un élément imprévu, des Acariens (environ deux ou trois pour toute la cellule de Nageotte). Le 21 septembre et, encore une fois, le 5 décembre, les Arthropodes sont retrouvés (un Acarien par $\mathrm{mm}^{3}$ environ). Le Laboratoire du $\mathrm{D}^{r} \mathrm{P}$. Aboudaram adresse ce dernier prélèvement au $\mathrm{P}^{\mathrm{r}} \mathrm{H}$. Harant, au Laboratoire d'Histoire naturelle médicule de la Faculté de Médecine de Montpellier. Nous reviendrons plus loin sur l'étude de ce liquide et sur les découvertes intéressantes qu'elle a permises.

A partir de cette date, l'état de la malade va en s'améliorant régulièrement et une nouvelle exploration rachidienne, le 12 janvier 1960 , est négative. A ce moment-là, les éléments blancs sont tombés à 6 par $\mathrm{mm}^{3}$ et l'albumine à $0,10 \mathrm{~g}$. La tension artérielle, à l'origine de $17 / 10$, était même descendue à $15 / 9$.

Mme L... a été revue depuis à plusieurs occasions, en mars pour l'ablation d'un kyste sébacé infecté du cuir chevelu, et en juillet pour une ponction lombaire de contrôle qui s'avère encore absolument normale.

En résumé, trois ponctions lombaires, pratiquées à une semaine, puis deux semaines d'intervalle, déconcertent les microscopistes par la présence de nombreux «Sarcoptes ». Il a été vérifié que la malade ne présentait pas de gale cutanée et que le matériel du médecin traitant était indemne de « parasites ». Le liquide céphalorachidien, auquel on a ajouté quelques gouttes de formol, parvient à Montpellier pour expertise.

Malgré les garanties prises plus haut, l'introduction accidentelle est immédiatement évoquée. Il ne fait aucun doute que ces pseudoparasites devaient se trouver sur le coton ou dans la lumière de l'aiguille ! Le fait en soi-même n'a rien d'étonnant puisque les Acariens, grâce à leur faible taille, peuvent entrer dans des tubes, même soigneusement bouchés au coton. D'après K. Samsinak, on a pu trouver Tyrophagus putrescentiæ dans des cultures de bactéries à l'Institut de Biologie de Prague.

Les aiguilles, laissées un long moment après la stérilisation, peuvent très bien avoir pu ètre contaminées. Malgré la répétition de l'incident, il n'y a vraisemblablement pas eu inoculation des Arthropodes, mais seulement leur entraînement par le liquide sortant des aiguilles provenant d'un même lot. La découverte, non pas d'une espèce, mais bien de deux espèces, dont l'une franchement saprophyte, devait emporter notre conviction. Nous allons même jusqu'à 
penser, avec $\mathrm{H}$. Harant, qu'à l'exception de quelques acariases respiratoires, contractées par inhalation, il n'existe aucun cas authentique de détermination viscérale confirmée.

Le liquide céphalo-rachidien étudié nous a montré plusieurs nymphes de Tarsonèmes à des stades différents et une deutonymphe du genre Suidasia.

\section{Tarsonemus hominis (Dahl).}

Le genre, non complètement recensé, comporte un grand nombre d'espèces vivant sur des végétaux divers, principalement exotiques (riz, thé, canne à sucre, ananas, etc...).

$T$. hominis, cosmopolite, se rencontre fréquemment dans les fleurs de Verbascum et est considéré comme un hôte domestique commun. Il existerait avec une particulière abondance dans le cho. colat. Dans des circonstances que nous connaissons mal, cet Acarien est susceptible de devenir un ectoparasite sous la peau des Mammifères et à la base des plumes de divers Oiseaux, spécialement des prédateurs.

Dahl, son découvreur, en 1910, et Saul l'ont trouvé dans des néoplasies humaines et animales, Blanc et Rollet (1910) dans les urines d'un malade présentant une cystite, et Carnegie Dickson (1920) dans les pièces d'autopsie d'un sujet décédé avec un gros foie et une polyadénopathie diffuse. Les urologues, avec Mackensie (1922), lui ont accordé une certaine constance dans un syndrome qui comporte énurésie nocturne, parfois hématurie, élimination de débris noirâtres, et présence au microscope de cellules épithéliales, d'Acariens entiers ou de débris (fragments de cuticule, pattes, poils). Dans sept cas évoquant des symptômes de cystite grave, l'origine interne a été évoquée parce que «les urines avaient été prélevées avec soin pour éliminer une contamination ». Il convient de noter qu'à côté de Tarsonemus hominis, il a été rencontré souvent Glyciphagus domesticus.

Patton et Evans vont jusqu'à suggérer que les Tarsonemoidea, capables dans la nature de percer la tige des plantes, "ont pu trouver leur chemin dans la vessie à travers la paroi rectale ». Il n'est pas besoin d'insister sur le caractère fantaisiste de cette assertion quand il est si simple de penser que, chez des malades recevant des ponctions ou des sondages fréquents, les Acariens sont, ou bien introduits par intervention humaine involontaire, ou bien entrainés à l'extérieur s'ils étaient dans la lumière des instruments de petite chirurgie... Il n'est pas sûr d'ailleurs qu'ils puissent proliférer dans les liquides organiques. Il se trouve que c'est surtout dans la vessie 
qu'ils peuvent être injectés par voie rétrograde, mais il manquait une observation d'Arthropodes dans le liquide céphalo-rachidien.

\section{Suidasia sp.}

Plus encore que le précédent, ce genre est très mal connu ! Il a été créé en 1905 par A.-C. Oudemans avec Suidasia pontifica, trouvé dans les tuyaux des rémiges de Araneus scolopaeus, un Oiseau de la région néotropicale.

Très petits et n'excédant jamais $400 \mu$, les Suidasia sont de découverte rarissime et leur étude est tout à fait débutante. Comme ils ont à la fois des affinités avec les Acarinæ et les Rhizogliphinæ, on a dû faire pour eux une sous-famille particulière, celle des Suidasinæ. Selon A.-A. Zachvatkin (1940), les Acaridæ comprendraient ainsi trois sous-familles : Acarinæ Ewing (= Tyrogliphinæ Zach), Suidasinæ Hughes et Rhizogliphinæ Zach.

La détermination exacte des Suidasia est, dans l'état actuel de la science, pratiquement impossible. Les spécialistes eux-mêmes, tel Oudemans, font preuve de beaucoup d'hésitations. C'est ainsi que Suidasia hamadryas Vitzthum est devenu, un an après sa découverte : Winterschmidtia hamadryas Oudemans; inversement, cet auteur avait décrit un Aphelenia qui fut ensuite ramené dans le genre Suidasia (c'était une nymphe ou peut-ètre même un mâle !)...

Les deux espèces, relativement les mieux connues du genre, sont Suidasia medanensis Oudemans et $S$. nesbitti Hughes. Jusqu'à présent, elles ont toujours été recueillies sur des matières organiques, jamais dans l'organisme de l'homme, ni des animaux. $S$. medanensis, trouvé dans un nid de Xylocope à Sumatra (Médan), n'a été revu que de rares fois, à notre connaissance, sur des cadavres d'Insectes à Porto-Rico, par I. Fox (1950), dans des cultures de Trogoderma granarium et sur des Pois importés du Nigéria, par A.-M. Hughes (1953). Mais, il s'avéra d'un entretien facile sur caséine à $22^{\circ}$ avec humidité de $87 \%$. Il a pu être noté que l'évolution totale ne dure que $16-18$ jours et que l'accouplement survient immédiatement après la dernière mue de la femelle. Quant à $S$. nesbitti, il a été décrit en 1949 par A.-M. Hughes dans une cargaison de farine de recoupe, en provenance d'Istamboul, et répartie entre Londres et Liverpool. Particulièrement abondant dans ce milieu, il semblait proliférer dans les conditions qui sont habituellement favorables à Tyroglyphus farinæ.

La deutonymphe isolée, qui a été trouvée dans le liquide céphalorachidien, mesure $125 \mu$. L'exemplaire monté, parfaitement identifiable quant au genre, est seulement dépourvu des pattes III et IV 

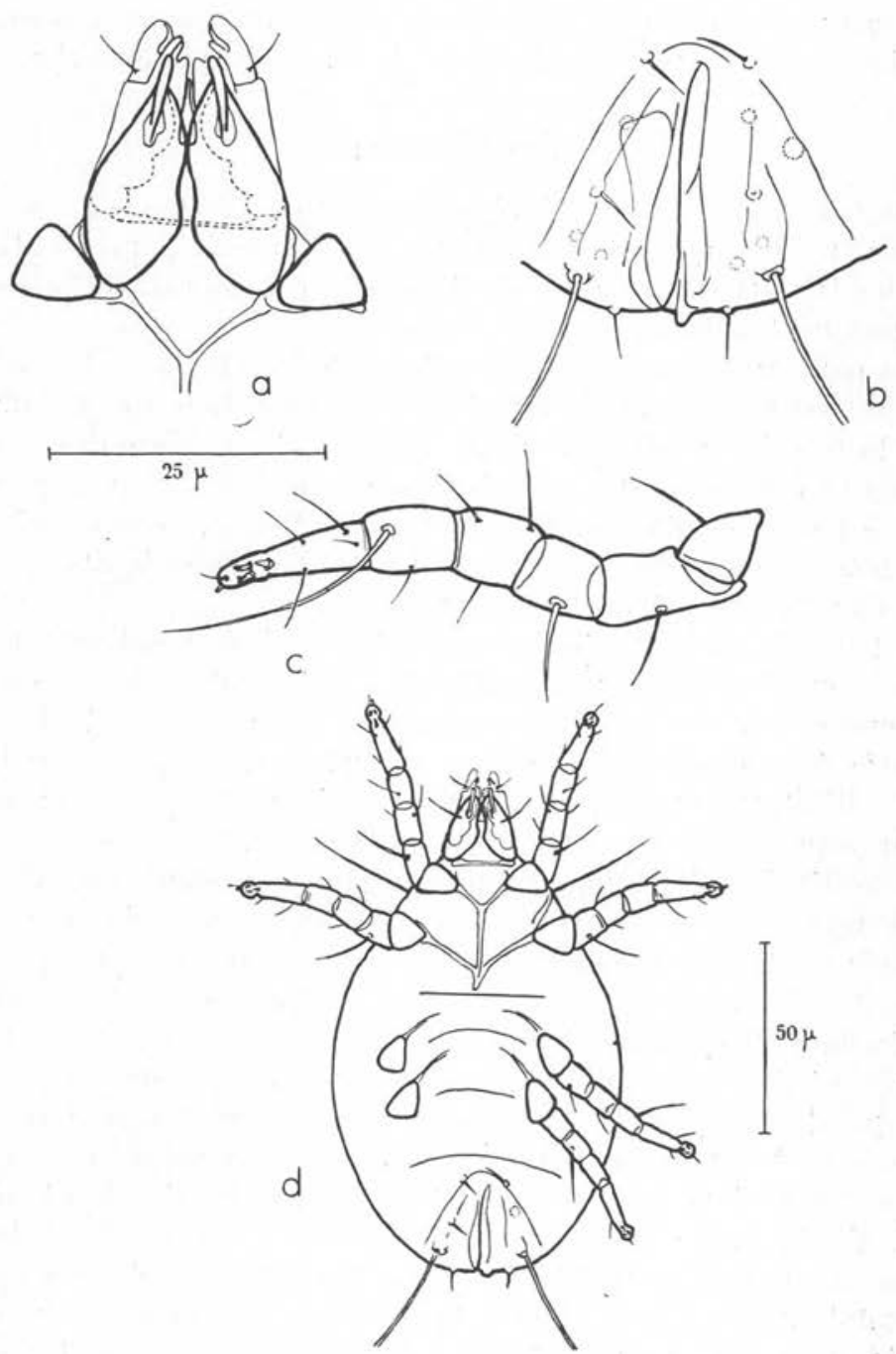

Suidasia $s p$. trouvé dans un liquide céphalo-rachidien. $a$, région céphalique; $b$, région anale; $c$, deuxième patte droite (vue ventrale); $d$, la deutonymphe vue en totalité.

droites. Ses caractères s'accordent assez bien avec ceux de Suidasia medanensis; la peau est finement ridée et non pas d'apparence écailleuse comme celle de $S$. nesbitti. Toutefois, nous ne pensons pouvoir nous prononcer catégoriquement sur sa diagnose, en présence d'un seul individu et dans un ètat immature. 
Nous osons croire en tout cas que cette observation méritait d'être rapportée, d'une part parce qu'elle parait faire justice des prétendues acariases de l'homme, d'autre part parce qu'elle apporte, à côté de la souillure secondaire presque classique par Tarsonemus hominis, la redécouverte parfaitement inopinée d'un Acarinæ saprophyte, connu de quelques rares régions chaudes du globe et appartenant au genre Suidasia.

\section{RÉSUMÉ}

Les auteurs rapportent une curieuse observation d'Acariens dans le liquide céphalo-rachidien chez une femme au Maroc. La souillure des aiguilles de ponction est certaine, malgré l'asepsie et les précautions habituellement prises. A trois reprises, le laboratoire de Casablanca retrouve ces Acariens (environ un par $\mathrm{mm}^{3}$ ). Deux genres sont déterminés: d'une part, Tarsonemus, presque classique, connu dans divers cas de pseudo-parasitisme ; d'autre part, Suidasia. Pour ce dernier, il s'agit d'une redécouverte parfaitement inopinée, car c'est un saprophyte des régions chaudes du globe qui n'a fait l'objet que de très rares observations.

\section{SUMMARY}

The authors relate an odd observation of Mites in the spinal fluid of a female patient in Morocco. The contamination of needles of lumbar punction is certain in spite of asepsy and usual precautions. Three times successively, the laboratory in Casablanca find Acarina (about one by $\mathrm{ml}$ ). Two genus are ascertained : Tarsonemus, almost classic, known by different cases of pseudoparasitism, and Suidasia. For the latter, the rediscovery is at least unexpected, because this Mite is a saprophyte of hot parts of the world and it was observed only at few occasions.

\section{Bibliographie SOMMAIRE}

(ne faisant état que des noms d'auteurs mentionnés dans le texte)

Blanc (G.) et Rollet (R.), 1910. - De la présence chez l'homme de Tarsonemus hominis Dahl. C.R. Soc. Biol. Paris, 69 (28), 233-235.

Carnegie Dickson (W. E.), 1921. - Mites as internal parasites of man. Journ. Tropic. Med. and Hyg., 24 (3), 25-27. 
DAнL (F. T.), 1910. - Milben als Erzeuger von Zellwucherungen. Centralbl. Bakteriol., I abt. 53 (5), 524-533.

Fox (I.), 1950. - A new tyroglyphid mite from Puerto Rico. Proc. Biol. Soc. Wash., 63, 205-208.

Hinst (A. S.), 1922. - Report on the mite (Tarsonemus floricolus Canestrini and Fanzago) found by Lt.-Col. J. Mackensie, R.A.M.C., in human urine. Journ. Roy. Arm. Med. Corps, 39 (5), 344-346.

Hughes (A. M.), 1949. - A new tyroglyphid mite, Suidasia nesbitti, sp. n. Proc. Zool. Soc. Lond., 118, 543-552.

- 1950. - On Suidasia medanensis Oudemans (Acaridiæ), a mite new to Great Britain. Proc. Zool. Soc. Lond., 125, 41-47.

MACKENSIE (J.), 1922. - Endoparasitic acari as a cause of urinary diseases. With notes on several cases. Journ. Roy. Med. Corps, 39 (5), 339-343.

Oudemans (A. C.), 1905. - Acarologische Aanteekeningen. Ent. Ber., 1, 209.

- 1924. - Acarologische Aanteekeningen. Ent. Ber., 6, 320.

Patton (IV. S.) et Evans (A. M.), 1939. - Insects, ticks, mites and venomous animals of medical and veterinary importance. Part. I : medical. Edit. H. R. Grubb Ltd, Croydon, 786 p., 374 fig.

SaUl (E.), 1910. - Milben in Geschwülsten. Deutsch. Med. Wchnschr., 36 (7), 338.

Zachvatkin (A. A.), 1940, - Acariens Tyroglyphoïdes. Edit. de l'Acad. des Sciences de l'U.R.S.S., Moscou, 474 p.

Laboratoires de Phytopathologie de l'Institut de Biologie de Prague

(Tchécoslovaquie) et d'Histoire naturelle médicale de la Faculté de Médecine de Montpellier 